\title{
Nrf2 activators as therapy for acute radiation dermatitis
} Yasuhiro Nakagami ${ }^{1,2}$

'Daiichi Sankyo Co., Ltd., 1-2-58 Hiromachi, Shinagawa-ku, Tokyo 140-8710, Japan

${ }^{2}$ Asubio Pharma Co., Ltd., 6-4-3 Minatojima-minamimachi, Chuo-ku, Kobe-shi, Hyogo 650-0047, Japan

Article Info

\section{Article Notes}

Received: April 13, 2017

Accepted: April 27, 2017

\section{${ }^{*}$ Correspondence:}

Yasuhiro Nakagami, Ph.D., Daiichi Sankyo Co., Ltd., 1-2-58, Hiromachi, Shinagawa-ku, Tokyo 140-8710, Japan, E-mail: nakagami.yasuhiro.y4@daiichisankyo.co.jp

(c) 2017 Yasuhiro Nakagami. This article is distributed under the terms of the Creative Commons Attribution 4.0 International License.

\section{Keywords}

Acute radiation dermatitis

Nrf2

RTA 402

RS9

\section{ABSTRACT}

Radiation therapy is a common treatment for cancer, often in combination with other approaches such as surgery and chemotherapy. High doses of radiation basically kill cancer cells; however, one of the common adverse effects of this treatment is acute radiation dermatitis. This leads to itching, pain, and diminished appearance, and can also interrupt the therapy itself. Reactive oxygen species are generated in the injured tissues, especially in keratinocytes, and cause inflammation, mitochondrial dysfunction, and DNA damage. Current drugs such as topical steroid creams and dressings are not sufficient to alleviate these detrimental events. Nuclear factor erythroid 2-related factor 2 (Nrf2) is a redox-sensitive transcription factor that binds to antioxidant response elements located in the promoter region of genes that encode antioxidant enzymes. Synthesized chemical Nrf2 activators have been shown to be effective in many pathological models, including acute radiation dermatitis models. Preclinical data identified RTA 402 as one of the most promising compounds for reducing or preventing acute radiation dermatitis, and this was recently tested in patients with breast cancer. This review discusses current links between Nrf2 activators and acute radiation dermatitis, and explores the possibility that symptoms can be alleviated by upregulating the Nrf2 signaling pathway.

\section{Acute radiation dermatitis and reactive oxygen species}

Radiation therapy is widely used to treat a variety of malignancies, and is often employed in conjunction with other approaches such as chemotherapy and tumor-removal surgery. The therapy basically kills cancer cells with curative or palliative purposes on specific areas of the body; however, the therapy can also cause damage to healthy cells. One of the most common side effects of radiation therapy is "acute" radiation dermatitis, which occurs in as many as $95 \%$ of patients who receive this treatment ${ }^{1,2}$. The pathology varies from mild erythema and dry desquamation to more severe moist desquamation and ulceration. These alterations diminish the skin's appearance and affect the patient's quality of life, and may also require that the radiation dose be limited or even that the therapy be interrupted. Moreover, loss of the skin barrier puts some patients at risk of bacterial infection. The development of "chronic" radiation dermatitis can occur from weeks to up to 10 years or more after the commencement of radiation, and the typical pathologic characteristics are fibrosis, telangiectasia, and loss of hair follicles. A number of approaches have been tested to help manage acute and chronic dermatitis, including topical steroid creams, nonsteroidal creams, dressings, and herbal medicines ${ }^{3}$, but there remain many 
unanswered questions and unfulfilled needs in this field. In this min-review, we fill focus on only acute radiation dermatitis because our preclinical data are mainly as to acute responses ${ }^{4}$.

Cells comprise about $80 \%$ water, and water radiolysis is therefore a critical consideration for radiation therapy. Absorption of ionizing radiation by water leads to the production of reactive oxygen species (ROS) in living cells ${ }^{5}$. It is well documented for many cell types that increased ROS disrupts nuclear DNA, either single or double strands, and leads to apoptosis. ROS also reacts quickly with nearby critical molecules, resulting in inflammatory cell recruitment, mitochondrial dysfunction, necrosis of skin cells and hypoxia induced by vascular endothelial damage ${ }^{1,6}$ (Figure 1). Furthermore, because these events are also triggers of ROS production, especially in keratinocytes ${ }^{7}$, it is often difficult to break the cycle of ROS-induced mediators that can cause radiation dermatitis. In an attempt to find ways to terminate this downward spiral, antioxidants were identified as a promising strategy for prophylaxis and treatment for some cases of acute radiation dermatitis ${ }^{8}$. Although many antioxidants, including polyphenols and pentoxifylline, have been tested, their use remains controversial ${ }^{3}$. The general limitations to their application include a weak potency for trapping or reducing continuously-generating ROS.

\section{Nrf2-Keap1 signaling and Nrf2 activators}

Nuclear factor erythroid 2-related factor 2 (Nrf2) is a basic leucine zipper (bZIP) transcription factor, which heterodimerizes with small Maf proteins and functions as a key player in the redox homeostatic gene regulatory network. Nrf2 is ubiquitously expressed, but expression is particularly high in tissues associated with detoxification (liver and kidneys) and those exposed to the external environment (skin, lungs, and gastrointestinal tract) 9 . $\mathrm{Nrf} 2$ is normally trapped within the cytosol by an adaptor protein, Kelch-like erythroid cell-derived protein with CNC homology (ECH)-associated protein 1 (Keap1) (Figure 1). It is rapidly degraded via the proteasomal pathway because Keap1 is an adaptor component of Cullin 3 (Cul3)based ubiquitin E3 ligase. Specific cysteine residues within Keap1 serve as primary sensors of stress signals, and their conformational modification inhibits Nrf2 ubiquitination ${ }^{10}$. Under stress conditions, stabilized Nrf2 is translocated to the nucleus, where it binds to a specific consensus ciselement, named the antioxidant-response element (ARE; also called the electrophile-responsive element (EpRE)). This element is present in the promoter region of genes that encode antioxidant and phase II detoxifying enzymes. There are over 250 Nrf2-targeted genes, including NAD(P) $\mathrm{H}$ :quinone oxidoreductase-1 (NQ01), heme oxygenase-1 (HO-1), glutamate cysteine ligase catalytic subunit (GCLC) and modifier subunit (GCLM), glutathione S-transferase, glutathione peroxidase, catalase, superoxide dismutase, and thioredoxin UDP-glucuronosyltransferase ${ }^{11}$. It is therefore expected that activation of Keap1-Nrf2 signaling will suppress ROS-induced inflammation and apoptosis ${ }^{10}$. In addition, potential crosstalk between the nuclear factor$\kappa \mathrm{B}(\mathrm{NF}-\kappa \mathrm{B})$ and Nrf2 pathways has been well studied ${ }^{12}$.

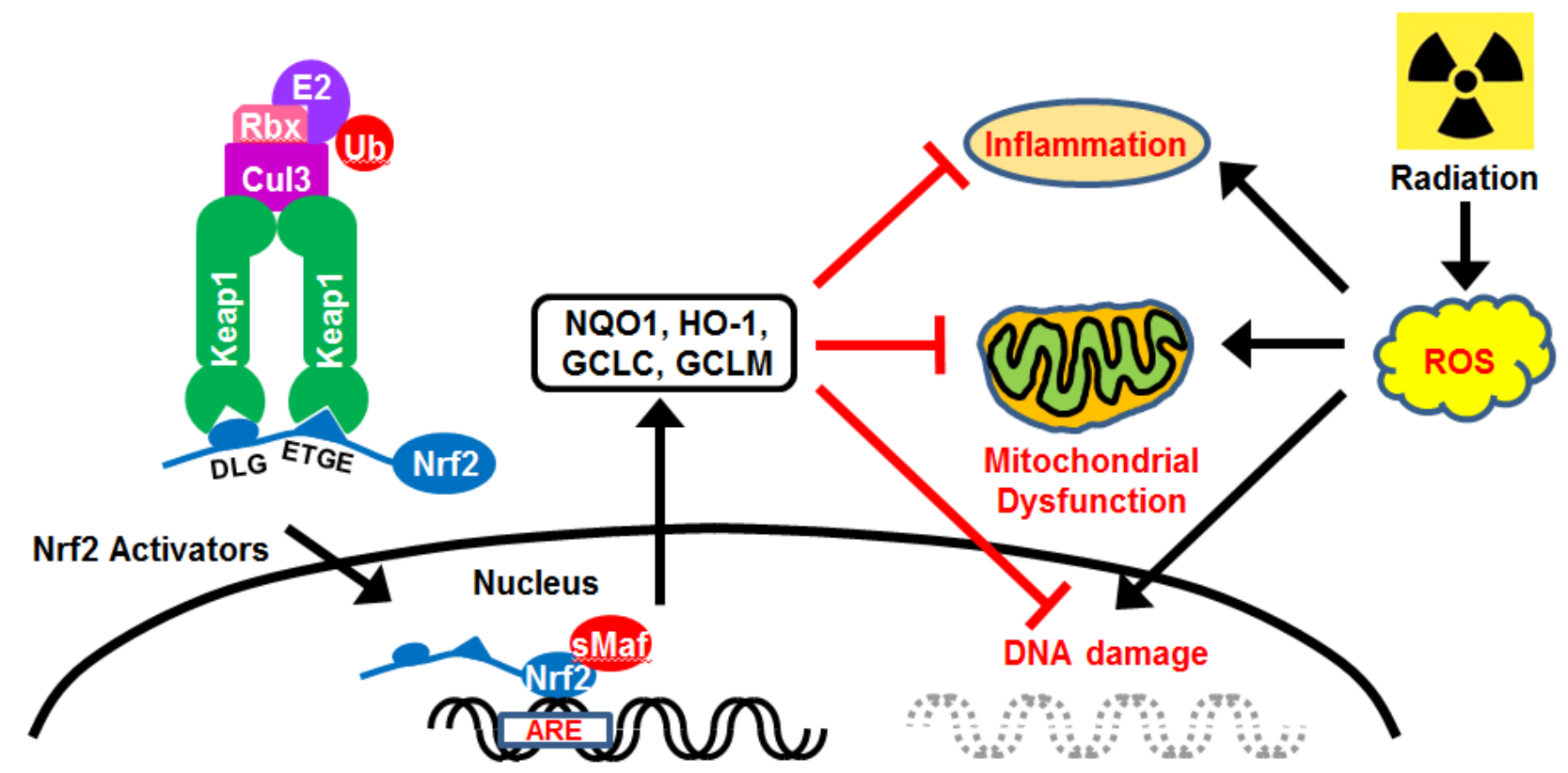

Figure 1: Keap1-Nrf2 signaling and ROS. The Keap1 homodimer binds to a single Nrf2 molecule via DLG and ETGE motifs, and Nrf2 is normally degraded by Cul3-dependent E3 ubiquitin ligase. Nrf2 activators accelerate the translocation of Nrf2 to the nucleus. Nrf2targeted antioxidant proteins alleviate ROS-induced inflammation, mitochondrial dysfunction, and DNA damage. 
(A)

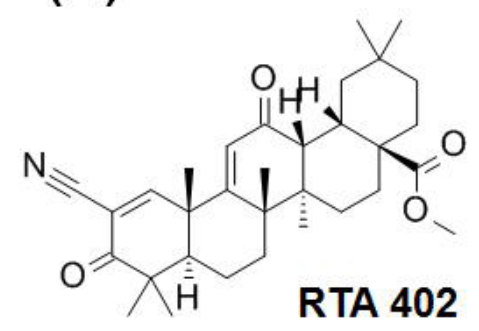

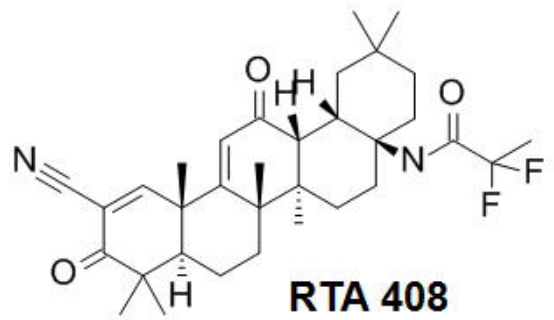

(B)

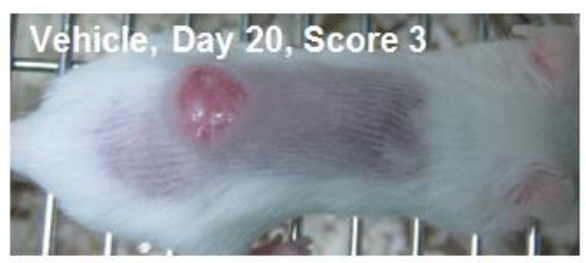

(C)

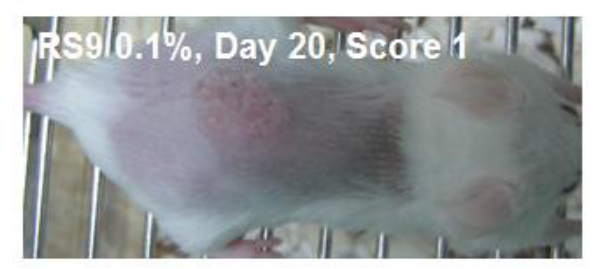

Figure 2: Nrf2 activators and acute radiation dermatitis. (A) Chemical structure of typical Nrf2 activators. (B and C) Macroscopic images of radiation dermatitis in vehicle or RS9-treated BALB/c mice ${ }^{4}$. Animals were irradiated with $30 \mathrm{~Gy} /$ day on Day 0 , and RS9 was topically applied to the back once daily from Day 1 to Day 30.

Activity of Nrf2 activators is generally determined by the concentration required to double NQ01 activity (CD) in murine Hepa1c1c7 hepatoma cells. The most potent Nrf2 activators are synthesized triterpenoids from oleanolic acid, which interact with cysteine residues of Keap1. The best known triterpenoid is bardoxolone methyl (2-cyano3,12-dioxooleana-1,9(11)-dien-28-oic acid (CDDO) methyl ester/CDDO-Me/RTA 402/bard) (Figure 2A), which has a CD value of approximately $1 \mathrm{nM}$, and is classified as an oral "antioxidant inflammation modulator"13. RTA 402 has undergone clinical testing for the treatment of many diseases, including chronic kidney disease ${ }^{14}$, Alport syndrome (ClinicalTrials.gov Identifier: NCT03019185), connective tissue disease associated pulmonary arterial hypertension (NCT02657356) and pulmonary hypertension (NCT02036970). Another derivative of RTA 408 , called omaveloxolone, has been tested in the treatment of radiation dermatitis (NCT02142959), post-surgical corneal endothelial cell loss (NCT02128113), Friedreich's ataxia (NCT02255435), mitochondrial myopathies (NCT02255422) and melanoma (NCT02259231). Because oxidative stress and inflammation are general phenomena in pathological conditions, it could be challenging to identify the most suitable diseases for Nrf2 activators.

\section{Nrf2 activators and acute radiation dermatitis}

Given the function of Nrf2-Keap1 signaling in stress responses, Nrf2 activators are a promising strategy for protecting skin cells in acute radiation dermatitis ${ }^{15,16}$. The efficacy of Nrf2 activators has been tested in the treatment of many diseases, mostly via oral administration, but adverse systemic effects are a big concern including a high cardiovascular mortality ${ }^{14}$. Therefore, topical application via a lotion or cream would be a preferred method, and would minimize adverse events of the treatment. Furthermore, it would be possible to restrict their use to only severely affected areas. Both preclinical and clinical data support not only the validity of this concept but also the safety of Nrf2 activators. Topical application of RTA 408 was shown to increase NQ01 and glutathione proteins in rat skin $^{7}$, and RTA 408 treatment resulted in a 55\% reduction in animal numbers with grade $\geq 2$ dermatitis scores compared to controls ${ }^{18}$. In the latter study, treatment with 1.0\% RTA 408 markedly suppressed epidermal thickening and dermal necrosis, and completely mitigated skin ulcers. In accordance with these improvements, an increase in the expression of Nrf2 target genes and a decrease in NF-kB target genes was observed. Moreover, oral administration of RTA 408 improved the efficacy of radiation therapy in mouse cancer models ${ }^{19}$. We also demonstrated that another Nrf2 activator, RS9 ${ }^{20,21}$, from microbial transformation, is effective at preventing acute radiation dermatitis in mice (Fig. 2B, C) ${ }^{4}$. The safety, pharmacokinetics, and local pharmacodynamics of RTA 408 were evaluated in a Phase 1 study (NCT02029716) 22 . Published data show that a twice-daily treatment with $0.5 \%$ and $3 \%$ RTA 408 lotion was well tolerated within a $500-\mathrm{cm}^{2}$ site for up to 28 days. The PRIMROSE study is a Phase 2 study that examines RTA 408 treatment in breast cancer patients at risk of radiation dermatitis (NCT02142959). The time-averaged effect on 
radiation dermatitis severity was set as the primary efficacy endpoint; however data regarding with progress of these trials are not available at present. Taking into account the tolerance of RTA 408, the results of the PRIMROSE study are highly anticipated.

\section{Conclusions}

Given our current understanding of the pathogenesis of acute radiation dermatitis, the use of antioxidants in the treatment of this condition is a highly rational approach. However, the effectiveness of typical antioxidants such as vitamins and calendula, which contain many polyphenolic antioxidants, must be investigated ${ }^{23}$. The accurate evaluation of drugs is challenging due to variations in patient populations, methodology, endpoints, agents studied, and moisturizing properties of the vehicle. A standardized classification system is therefore required for testing new drugs in well-designed clinical trials ${ }^{24}$. While rodent systems are commonly used in current preclinical studies, it is important to note that radiation-induced skin responses in mice require double the dose compared to humans ${ }^{25}$. The advantage of pigs should be considered if we are to accurately extrapolate the safety and efficacy of drugs, because the skin structure of pigs is similar to that of humans ${ }^{26,27}$.

Nrf2 is a master regulator of antioxidant defense genes, suggesting that Nrf2 activators may be able to fully suppress radiation/ROS-induced events in skin injuries by inducing a vast array of our body's important antioxidants ${ }^{28,29}$. However, the etiology of skin injury is not simple; therefore, additional intervention may be necessary, especially in the case of chronic radiation dermatitis. In this stage, a complex cascade of detrimental events, including pain, fibrosis, telangiectasia, necrosis, and ulcer, can occur. In addition, the activation of Nrf2 is a double-edged sword because it might make cancer cells resistant to radiation and anticancer drugs ${ }^{10,30}$. Therefore, the contribution of Nrf2 and other molecules should be further investigated in the context of each condition in order to open up new directions of research for strategies to cure not only radiation dermatitis but also other ROS-related diseases.

\section{Acknowledgements}

This work was supported by Daiichi Sankyo Co., Ltd. and Asubio Pharma Co., Ltd.

\section{References}

1. Ryan JL. Ionizing radiation: the good, the bad, and the ugly. J Invest Dermatol. 2012; 132: 985-993.

2. Kutner A, Makdisi J, Friedman A. Radiation dermatitis: current perspectives and future directions. J Drugs Dermatol. 2012; 11:, 1127-1131.

3. Salvo N, Barnes E, van Draanen J, et al. Prophylaxis and management of acute radiation-induced skin reactions: a systematic review of the literature. Curr Oncol. 2010; 17: 94-112.
4. Nakagami Y, Masuda K. A novel Nrf2 activator from microbial transformation inhibits radiation-induced dermatitis in mice. J Radiat Res. 2016; 57: 567-571.

5. Azzam EI, Jay-Gerin JP, Pain D. Ionizing radiation-induced metabolic oxidative stress and prolonged cell injury. Cancer Lett. 2012; 327: 4860.

6. Singh M, Alavi A, Wong R, et al. Radiodermatitis: a review of our current understanding. Am J Clin Dermatol. 2016; 17: 277-292.

7. Bito $T$, Nishigori C. Impact of reactive oxygen species on keratinocyte signaling pathways. J Dermatol Sci. 2012; 68: 3-8.

8. Amber KT, Shiman MI, Badiavas EV. The use of antioxidants in radiotherapy-induced skin toxicity. Integr Cancer Ther. 2014; 13: 3845 .

9. Itoh K, Mimura J, Yamamoto M. Discovery of the negative regulator of Nrf2, Keap1: a historical overview. Antioxid Redox Signal. 2010; 13: $1665-1678$

10. Suzuki T, Motohashi H, Yamamoto M. Toward clinical application of the Keap1-Nrf2 pathway. Trends Pharmacol Sci. 2013; 34: 340-346.

11. Ruiz S, Pergola PE, Zager RA, et al. Targeting the transcription factor Nrf2 to ameliorate oxidative stress and inflammation in chronic kidney disease. Kidney Int. 2013; 83: 1029-1041.

12. Wardyn JD, Ponsford AH, Sanderson CM. Dissecting molecular crosstalk between Nrf2 and NF- $\mathrm{kB}$ response pathways. Biochem Soc Trans. 2015; 43: 621-626.

13. Liby KT, Sporn MB. Synthetic oleanane triterpenoids: multifunctional drugs with a broad range of applications for prevention and treatment of chronic disease. Pharmacol Rev. 2012; 64: 972-1003.

14. Abboud HE. Synthetic oleanane triterpenoids: magic bullets or not? Kidney Int. 2013; 83: 785-787.

15. Greenwald MB, Ben Sasson S, Bianco Peled H, et al. Skin redox balance maintenance: the need for an Nrf2-activator delivery system. Cosmetics. 2016; 3: 1.

16. Gęgotek A, Skrzydlewska E. The role of transcription factor Nrf2 in skin cells metabolism. Arch Dermatol Res. 2015; 307: 385-396.

17. Reisman SA, Lee CY, Meyer CJ, et al. Topical application of the synthetic triterpenoid RTA 408 activates Nrf2 and induces cytoprotective genes in rat skin. Arch Dermatol Res. 2014; 306: 447-454.

18. Reisman SA, Lee CY, Meyer CJ, et al. Topical application of the synthetic triterpenoid RTA 408 protects mice from radiation-induced dermatitis. Radiat Res. 2014; 181: 512-520.

19. Alexeev V, Lash E, Aguillard A, et al. Radiation protection of the gastrointestinal tract and growth inhibition of prostate cancer xenografts by a single compound. Mol Cancer Ther. 2014; 13: 29682977.

20. Nakagami Y, Masuda K, Hatano E, et al. Novel Nrf2 activators from microbial transformation products inhibit blood-retinal barrier permeability in rabbits. Br J Pharmacol. 2015; 172: 1237-1249.

21. Nakagami Y. Nrf2 is an attractive therapeutic target for retinal diseases. Oxid Med Cell Longev. 2016; 7469326.

22. Reisman SA, Goldsberry AR, Lee CY, et al. Topical application of RTA 408 lotion activates Nrf2 in human skin and is well-tolerated by healthy human volunteers. BMC Dermatol. 2015; 15:10.

23. Kodiyan J, Amber KT. A review of the use of topical Calendula in the prevention and treatment of radiotherapy-induced skin reactions. Antioxidants. 2015; 4: 293-303.

24. Chen AP, Setser A, Anadkat MJ, et al. Grading dermatologic adverse events of cancer treatments: the Common Terminology Criteria for Adverse Events Version 4.0. J Am Acad Dermatol. 2012; 67: 10251039. 
25. Williams JP, Brown SL, Georges GE, et al. Animal models for medical countermeasures to radiation exposure. Radiat Res. 2010; 173: 557-578.

26. van den Aardweg GJ, Hopewell JW, Guttenberger R. The kinetics of repair of sublethal radiation-induced damage in pig skin: studies with multiple interfraction intervals. Radiat Res. 1996; 145: 586-594.

27. Mahl JA, Vogel BE, Court M, et al. The minipig in dermatotoxicology: methods and challenges. Exp Toxicol Pathol. 2006; 57: 341-345.
28. Gorrini C, Harris IS, Mak TW. Modulation of oxidative stress as an anticancer strategy. Nat Rev Drug Discov. 2013; 12: 931-947.

29. Schäfer M, Werner S. Nrf2 A regulator of keratinocyte redox signaling. Free Radic Biol Med. 2015; 88: 243-252.

30. Milkovic L, Zarkovic N, Saso L. Controversy about pharmacological modulation of Nrf2 for cancer therapy. Redox Biol. 2017; 12: 727-732. 